\title{
Progressive loss of supination of the wrist
}

\author{
Amandine Crombé ${ }^{1}$ - Yacine Carlier ${ }^{2}$ François Le Loarer ${ }^{3} \cdot$ Benjamin Dallaudiere $^{4}$. \\ Lionel Pesquer ${ }^{4}$
}

Published online: 31 July 2017

(C) ISS 2017

\section{Question}

HISTORY: A 46-year-old male had progressive and complete loss of supination of the right wrist, without pain or prior trauma. Normal radiographs and CT arthrography of the wrist were taken. Ultrasonography (Fig. 1) followed by MRI was performed (Fig. 2) as was a US-guided biopsy (Fig. 3).

The diagnosis can be found at doi:10.1007/s00256-017-2722-6

Amandine Crombé

amandine.crombe@ens-lyon.fr

1 Department of Radiology, Institut Bergonié, 229 cours de l'Argonne, 33076 Bordeaux Cedex, France

2 Department of Orthopedic Surgery, Clinique du sport de Bordeaux-Merignac, 2 rue Négrevergne, 33700 Mérignac, France

3 Department of Pathology, Institut Bergonié, 229 cours de l'Argonne, 33076 Bordeaux Cedex, France

4 Centre d'imagerie ostéo-articulaire, Clinique du sport de Bordeaux-Mérignac, 2 rue Négrevergne, 33700 Mérignac, France 


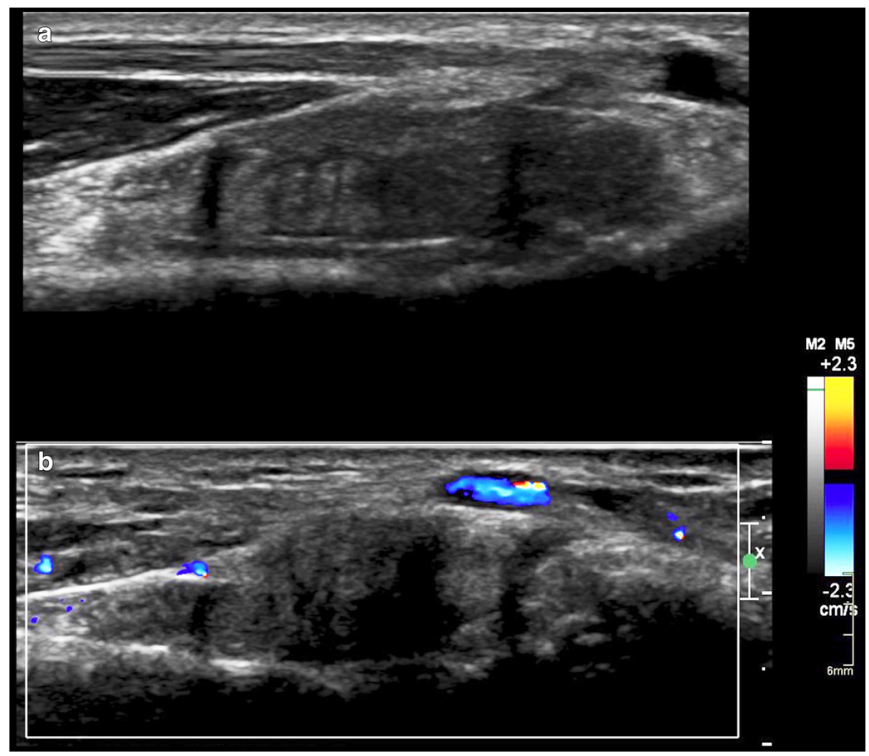

Fig. 1 Longitudinal slice of the anterior side of the right wrist (a) with Doppler (b).
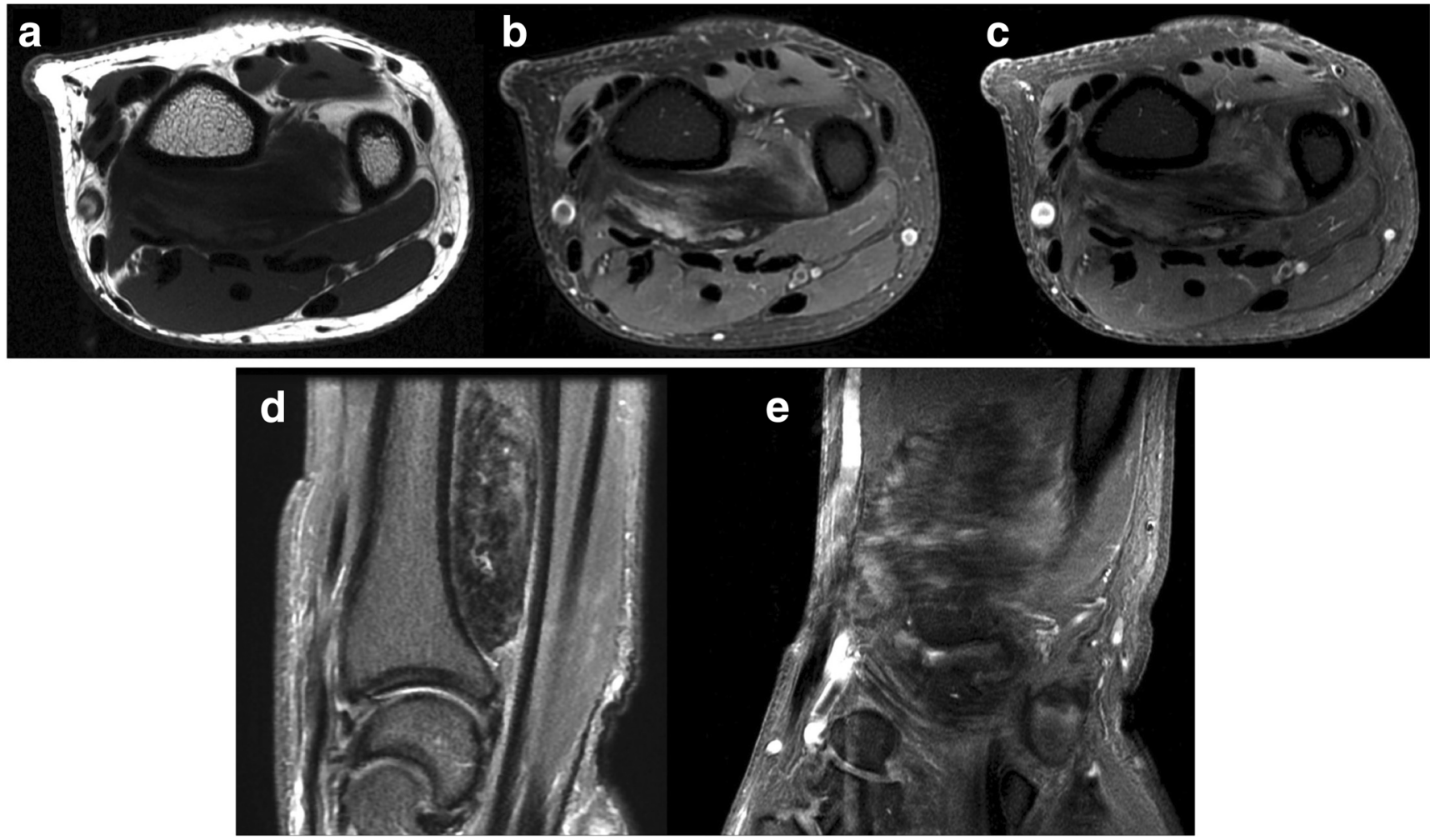

Fig. 2 T1-weighted MRI (a). Fat-suppressed proton density-weighted MRI (b). Fat-suppressed T1-weighted MRI after gadolinium chelate injection (c, d) 

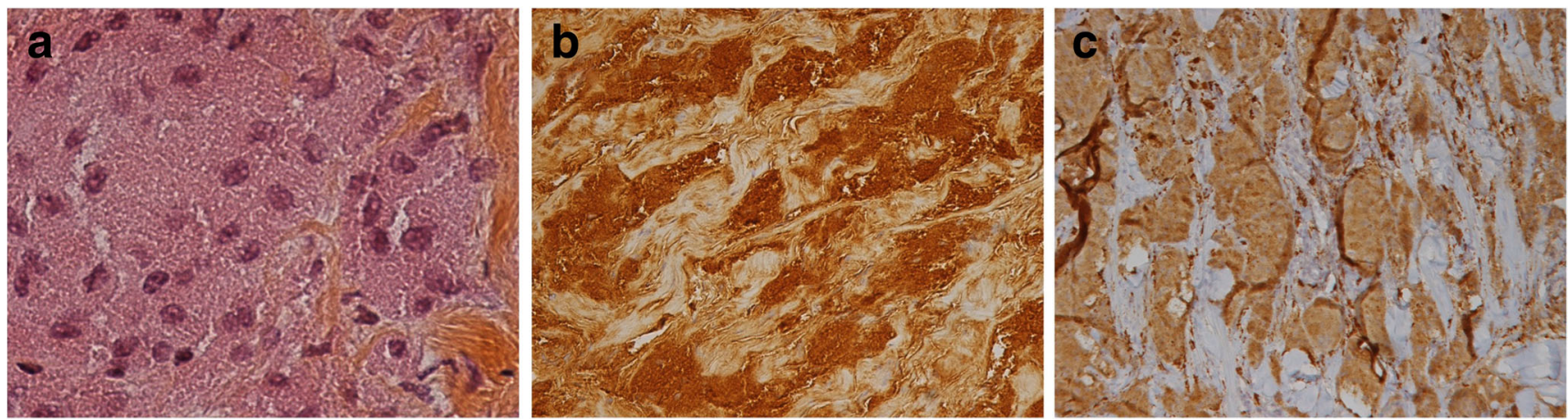

Fig. 3 Hematoxylin-eosin saffron staining (magnification $\times 100)($ a). Immunohistostains including S100-protein $(\mathbf{b})$ and CD-68 (c)

\section{Compliance with ethical standards}

Funding No funding was received for this article.
Conflict of interest The authors declare that they have no conflicts of interest. 